\title{
A note on the IBVP for wave equations with dynamic boundary conditions
}

\section{Chan Li and Ti-Jun Xiao*}

\section{"Correspondence:}

tjxiao@fudan.edu.cn

Shanghai Key Laboratory for

Contemporary Applied

Mathematics, School of

Mathematical Sciences, Fudan

University, Shanghai, 200433,

P.R. China

\begin{abstract}
In this paper, we investigate the controllability on the IBVP for a class of wave equations with dynamic boundary conditions by the HUM method as well as the wellposedness for the related back-ward problems. After proving a new observability inequality, we establish new wellposedness and controllability theorems for the IBVP.
\end{abstract}

Keywords: Wentzell boundary condition; wave equation; wellposedness; controllability

\section{Introduction}

In this paper, we consider the exact boundary controllability on the IBVP for wave equation with dynamic boundary condition as follows:

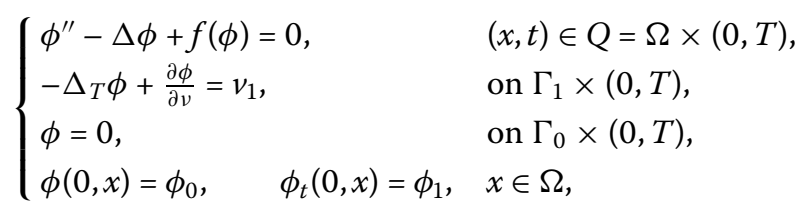

where $\Omega \subset \mathbb{R}^{n}$ is a bounded domain with smooth boundary $\Gamma_{0} \cup \Gamma_{1}, \bar{\Gamma}_{0} \cap \bar{\Gamma}_{1}=\emptyset$, and $\Delta_{T}$ is tangential Laplace operator. The boundary condition on $\Gamma_{1}$ is called the static Wentzell boundary condition and the dynamic Wentzell boundary condition is

$$
\phi^{\prime \prime}-\Delta_{T} \phi+\frac{\partial \phi}{\partial v}=v_{1}, \quad \text { on } \Gamma_{1} \times(0, T)
$$

The system models an elastic body's transverse vibration. For details, please see the paper of Lemrabet [1]. In [1-7] and the references therein, one can find more details as regards dynamic boundary conditions. Moreover, Heminna [3] gives the controllability for elasticity system with two controls: both tangential and normal, under the assumption of the wellposedness for the backward system, which is a key assumption for getting controllability. In this paper, we establish first of all the wellposedness theorem for back-ward systems based on the transposition method ( $c f$. [8]) and then obtain the controllability on the IBVP for the wave equation above by using the method of HUM.

(c) $2016 \mathrm{Li}$ and Xiao. This article is distributed under the terms of the Creative Commons Attribution 4.0 International License (http://creativecommons.org/licenses/by/4.0/), which permits unrestricted use, distribution, and reproduction in any medium, provided you give appropriate credit to the original author(s) and the source, provide a link to the Creative Commons license, and indicate if changes were made. 


\section{Boundary controllability for Wentzell systems}

For simplicity, we write

$$
V=H_{\Gamma_{0}}^{1}(\Omega):=\left\{v \in H^{1}(\Omega):\left.v\right|_{\Gamma_{1}} \in H^{1}\left(\Gamma_{1}\right),\left.v\right|_{\Gamma_{0}}=0\right\}, \quad \mathcal{H}=V \times L^{2}(\Omega),
$$

with the norm

$$
\begin{aligned}
& \|u\|_{V}^{2}=\|\nabla u\|_{L^{2}(\Omega)}^{2}+\left\|\nabla_{T} u\right\|_{L^{2}\left(\Gamma_{1}\right)}^{2}, \\
& \|(u, v)\|_{\mathcal{H}}^{2}=\|u\|_{V}^{2}+\|v\|_{L^{2}(\Omega)}^{2} .
\end{aligned}
$$

We study the controllability under the geometric condition:

$$
\exists x_{0} \in \mathbb{R}^{n}, \quad\left(x-x_{0}\right) \cdot v \leq 0, \quad \text { on } \Gamma_{0} .
$$

Take a look at the linear homogeneous system first,

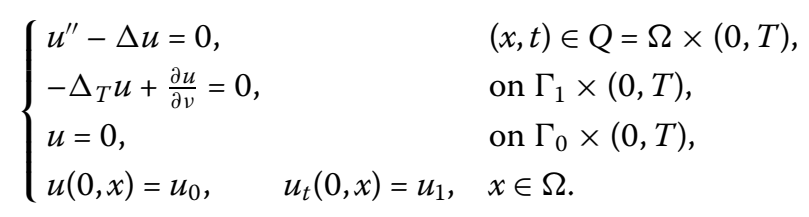

The wellposedness for the problem (2.1) is not hard to see. Define an operator $\mathcal{A}: D(\mathcal{A}) \rightarrow$ $\mathcal{H}$ by

$$
\mathcal{A}\left(\begin{array}{l}
u \\
v
\end{array}\right):=\left(\begin{array}{c}
v \\
\Delta u
\end{array}\right),
$$

with

$$
\begin{aligned}
& D(\mathcal{A}):=\left\{(u, v) \in \mathcal{H}: \Delta u \in L^{2}(\Omega), v \in V, \partial_{\nu} u-\Delta_{T} u=0\right\}, \\
& D\left(\mathcal{A}^{2}\right)=\left\{(u, v)^{T} \in D(\mathcal{A}): \mathcal{A}(u, v)^{T} \in \mathcal{H}\right\} .
\end{aligned}
$$

Write

$$
E(t):=\frac{1}{2} \int_{\Omega}\left(|\nabla u|^{2}+\left|u^{\prime}\right|^{2}\right) d x+\frac{1}{2} \int_{\Gamma_{1}}\left|\nabla_{T} u\right|^{2} d s .
$$

Then it is clear that $E(t)=E(0)$.

Lemma 2.1 (Observability inequality) For $T>2 R$,

$$
E(0) \leq C \int_{\Sigma_{1}}\left(u^{\prime 2}+u^{2}+\left|\nabla_{T} u\right|^{2}+\left|\Delta_{T} u\right|^{2}\right) d s d t
$$

where $R=\max _{x \in \bar{\Omega}}\left|x-x_{0}\right|, \Sigma_{1}=(0, T) \times \Gamma_{1}$. 
Proof Multiply the equation with the radial multiplier $\left(x-x_{0}\right) \cdot \nabla u+\frac{n-1}{2} u$ and integrate by parts in $Q$. Then we obtain

$$
\begin{aligned}
& \frac{1}{2} \int_{Q}\left(\left|u^{\prime}\right|^{2}+|\nabla u|^{2}\right) d x d t+\frac{1}{2} \int_{\Sigma_{1}}\left|\nabla_{T} u\right|^{2} d s d t+\mid\left\langle u^{\prime},\left(x-x_{0}\right) \cdot \nabla u+\left.\frac{n-1}{2} u\right|_{0} ^{T}\right. \\
& =\frac{1}{2} \int_{\Sigma_{1}}\left(x-x_{0}\right) \cdot v\left|u^{\prime}\right|^{2} d s d t+\int_{\Sigma_{1}} \frac{\partial u}{\partial v}\left(x-x_{0}\right) \cdot \nabla u d s d t \\
& \quad+\frac{n-1}{2} \int_{\Sigma_{1}} u \frac{\partial u}{\partial v} d s d t+\frac{1}{2} \int_{0}^{T} \int_{\Gamma_{0}}\left(x-x_{0}\right) \cdot v\left|\frac{\partial u}{\partial v}\right|^{2} d s d t \\
& \quad+\frac{1}{2} \int_{\Sigma_{1}}\left(\left|\nabla_{T} u\right|^{2}-\left(x-x_{0}\right) \cdot v|\nabla u|^{2}\right) d s d t .
\end{aligned}
$$

It is easy to see that

$$
\left|\left\langle u^{\prime},\left(x-x_{0}\right) \cdot \nabla u+\frac{n-1}{2} u\right\rangle\right|_{0}^{T} \leq 2 R E(0)+c(T) \int_{\Sigma_{1}}\left(u^{2}+u^{\prime 2}\right) d s d t .
$$

Combining with the geometric condition $\left(x-x_{0}\right) \cdot v \leq 0$ on $\Gamma_{0}$, we deduce from (2.3) and (2.1) that

$$
\begin{aligned}
(T-2 R) E_{0} \leq & c_{1} \int_{\Sigma_{1}}\left|u^{\prime}\right|^{2} d s d t+\int_{\Sigma_{1}} \frac{\partial u}{\partial v}\left(x-x_{0}\right) \cdot \nabla u d s d t \\
& +c(T) \int_{\Sigma_{1}} u^{2} d s d t+\frac{n-1}{2} \int_{\Sigma_{1}} u \frac{\partial u}{\partial v} d s d t+\frac{1}{2} \int_{\Sigma_{1}}\left|\nabla_{T} u\right|^{2} d s d t \\
\leq & c \int_{\Sigma_{1}}\left(\left|u^{\prime}\right|^{2}+\left|\Delta_{T} u\right|^{2}+u^{2}+\left|\nabla_{T} u\right|^{2}\right) d s d t .
\end{aligned}
$$

So, the observability inequality (2.2) holds.

The observability inequality (2.2) enables us to define the following norm:

$$
\left\|\left(u_{0}, u_{1}\right)\right\|_{F}^{2}:=\int_{\Sigma_{1}}\left(\left|u^{\prime}\right|^{2}+\left|\Delta_{T} u\right|^{2}+u^{2}+\left|\nabla_{T} u\right|^{2}\right) d s d t
$$

and the corresponding inner product

$$
\left\langle\left(u_{0}, u_{1}\right),\left(v_{0}, v_{1}\right)\right\rangle_{F}:=\int_{\Sigma_{1}}\left(u^{\prime} v^{\prime}+\Delta_{T} u \Delta_{T} v+u v+\nabla_{T} u \nabla_{T} v\right) d s d t
$$

where $u$ (or $v)$ is the solution of (2.1) with initial data $\left(u_{0}, u_{1}\right)$ (or $\left.\left(v_{0}, v_{1}\right)\right)$. Let

$$
F:={\overline{\left\{\left(u_{0}, u_{1}\right) \in C^{\infty}(\bar{\Omega}) \times C^{\infty}(\bar{\Omega}): \partial_{\nu} u_{0}-\Delta_{T} u_{0}=0\right.}}^{\|\cdot\|_{F}} .
$$

Then $\left(F,\langle\cdot, \cdot\rangle_{F}\right)$ is a Hilbert space.

Now we consider the wellposedness for the linear backward problem

$$
\begin{cases}\phi^{\prime \prime}-\Delta \phi=0, & \text { in } Q, \\ \frac{\partial \phi}{\partial v}-\Delta_{T} \phi=v, & \text { on } \Gamma_{1} \times(0, T), \\ \phi=0, & \text { on } \Gamma_{0} \times(0, T),\end{cases}
$$


with terminal data

$$
\phi(T)=\phi_{0}, \quad \phi^{\prime}(T)=\phi_{1}, \quad \text { in } \Omega,
$$

where

$$
v(x, t)=-\partial_{t} u^{\prime}+\Delta_{T}\left(\Delta_{T} u\right)-\Delta_{T} u+u
$$

and $\partial_{t}$ is taken in the following sense:

$$
\left\langle-\partial_{t} u^{\prime}, \psi\right\rangle=\left\langle u^{\prime}, \psi^{\prime}\right\rangle, \quad \forall \psi \in H^{1}\left(0, T ; L^{2}(\Omega)\right)
$$

For every

$$
\left(\theta, \theta^{\prime}\right) \in C\left((0, T+\varepsilon) ; D\left(\mathcal{A}^{2}\right)\right) \cap C^{1}((0, T+\varepsilon) ; D(\mathcal{A})) \cap C^{2}((0, T+\varepsilon) ; \mathcal{H})
$$

with $\theta(0)=\theta^{\prime}(0)=0$, we say $\phi \in L^{\infty}\left(0, T ; V^{\prime}\right)$ is the solution of (2.5)-(2.6) if it satisfies the following equality:

$$
\begin{gathered}
\int_{Q} \phi f d Q+\left\langle\phi^{\prime}(T), \theta(T)\right\rangle_{F^{\prime}, F}-\left\langle\phi(T), \theta^{\prime}(T)\right\rangle_{F^{\prime}, F} \\
=-\int_{\Sigma_{1}}\left(\nabla_{T} u \nabla_{T} \theta+\Delta_{T} u \Delta_{T} \theta+u^{\prime} \theta^{\prime}+u \theta\right) d s d t,
\end{gathered}
$$

where

$$
f=\theta^{\prime \prime}-\Delta \theta \in L^{1}(0, T ; V)
$$

It is clear that $\theta$ satisfies

$$
\begin{cases}\theta^{\prime \prime}-\Delta \theta=f, & \text { in } Q, \\ \frac{\partial \theta}{\partial \nu}-\Delta_{T} \theta=0, & \text { on } \Gamma_{1}, \\ \theta=0, & \text { on } \Gamma_{0}, \\ \theta(0)=0, \quad \theta^{\prime}(0)=0, & \text { in } \Omega .\end{cases}
$$

Theorem 2.2 In the sense of (2.7), the problem (2.5)-(2.6) has a unique solution $\phi$ satisfying

$$
\phi \in L^{\infty}\left(0, T ; V^{\prime}\right)
$$

Proof First of all, we give the energy estimate for the nonhomogeneous system (2.8).

For the general energy (the low-order energy), since

$$
\frac{1}{2} \frac{d}{d t}\left(\int_{\Omega} \theta^{\prime 2}+|\nabla \theta|^{2} d x+\int_{\Gamma_{1}}\left|\nabla_{T} \theta\right|^{2} d s\right)=\int_{\Omega} f \theta_{t} d x
$$

and

$$
E(T)=E(t)+\int_{t}^{T} \int_{\Omega} f \theta^{\prime} d x d t
$$


we have

$$
E(t) \leq C_{T}\left(E(T)+\|f\|_{L^{2}\left(0, T ; L^{2}(\Omega)\right)}^{2}\right), \quad \forall t \in(0, T) .
$$

For the high-order energy, we have

$$
E_{1}(t)=\frac{1}{2} \int_{\Omega}\left|\nabla \theta^{\prime}\right|^{2}+|\Delta \theta|^{2} d x+\frac{1}{2} \int_{\Gamma_{1}}\left|\nabla_{T} \theta^{\prime}\right|^{2} d s
$$

and

$$
E_{1}(T)=E_{1}(t)+\int_{t}^{T} \int_{\Omega} f \Delta \theta^{\prime} d x d t .
$$

Hence,

$$
\begin{aligned}
E_{1}(t)= & E_{1}(T)+\int_{t}^{T} \int_{\partial \Omega} f \frac{\partial \theta^{\prime}}{\partial v} d s d t-\int_{t}^{T} \int_{\Omega} \nabla f \nabla \theta^{\prime} d x d t \\
= & E_{1}(T)+\int_{t}^{T} \int_{\Gamma_{1}} f \Delta_{T} \theta^{\prime} d s d t-\int_{t}^{T} \int_{\Omega} \nabla f \nabla \theta^{\prime} d x d t \\
\leq & E_{1}(T)+\int_{t}^{T}\left(\int_{\Gamma_{1}}\left\|\nabla_{T} f\right\|^{2} d s\right)^{\frac{1}{2}}\left(\int_{\Gamma_{1}}\left|\nabla_{T} \theta^{\prime}\right|^{2} d s\right)^{\frac{1}{2}} d t \\
& +\int_{t}^{T}\left(\int_{\Omega}|\nabla f|^{2} d x\right)^{\frac{1}{2}}\left(\int_{\Omega}\left|\nabla \theta^{\prime}\right|^{2} d x\right)^{\frac{1}{2}} d t \\
\leq & E_{1}(T)+\left\|E_{1}(t)\right\|_{L^{\infty}(0, T)}^{\frac{1}{2}}\|f\|_{L^{1}(0, T ; V)},
\end{aligned}
$$

which implies that

$$
E_{1}(t) \leq C\left(E_{1}(T)+\|f\|_{L^{1}(0, T ; V)}^{2}\right), \quad 0 \leq t \leq T .
$$

Let $\theta=\theta_{1}+\theta_{2}$, where $\theta_{1}$ satisfies

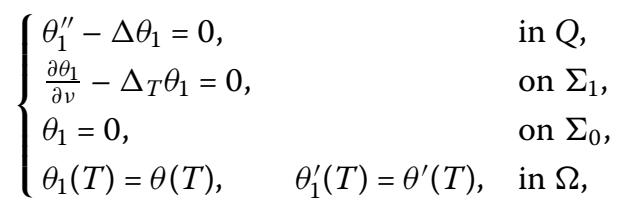

and $\theta_{2}$ satisfies

$$
\begin{cases}\theta_{2}^{\prime \prime}-\Delta \theta_{2}=f, & \text { in } Q, \\ \frac{\partial \theta_{2}}{\partial \nu}-\Delta_{T} \theta_{2}=0, & \text { on } \Sigma_{1} \\ \theta_{2}=0, & \text { on } \Sigma_{0} \\ \theta_{2}(T)=0, \quad \theta_{2}^{\prime}(T)=0, & \text { in } \Omega .\end{cases}
$$

Let

$$
L\left(\theta(T), \theta^{\prime}(T), f\right)=\int_{\Sigma_{1}}\left(\nabla_{T} u \nabla_{T} \theta+\Delta_{T} u \Delta_{T} \theta+u_{t} \theta_{t} d+u \theta\right) d s d t .
$$


Then we obtain

$$
\begin{aligned}
& L\left(\theta(T), \theta^{\prime}(T), f\right) \\
& =\int_{\Sigma_{1}}\left(\nabla_{T} u \nabla_{T} \theta+\Delta_{T} u \Delta_{T} \theta+u_{t} \theta_{t}+u \theta\right) d s d t \\
& \leq \int_{\Sigma_{1}}\left(\nabla_{T} u \nabla_{T} \theta_{1}+\Delta_{T} u \Delta_{T} \theta_{1}+u_{t} \theta_{1 t}+u \theta_{1}\right. \\
& \left.\quad+\nabla_{T} u \nabla_{T} \theta_{2}+\Delta_{T} u \Delta_{T} \theta_{2}+u^{\prime} \theta_{2}^{\prime}+u \theta_{2}\right) d s d t \\
& \leq C\left(\|\{\theta(T), \theta(T)\}\|_{F}^{2}+\|f\|_{L^{1}(0, T ; V)}^{2}\right)^{\frac{1}{2}} .
\end{aligned}
$$

Therefore, $L: F \times L^{1}(0, T ; V) \rightarrow L^{\infty}\left(0, T ; V^{\prime}\right) \times F^{\prime}$ is a bounded operator. So $\exists \phi \in$ $L^{\infty}\left(0, T ; V^{\prime}\right),\left(\rho_{1},-\rho_{0}\right) \in F^{\prime}$ such that

$$
\begin{aligned}
& \int_{Q} \phi f d x d t-\left\langle\rho_{1}, \theta(T)\right\rangle+\left\langle\rho_{0}, \theta^{\prime}(T)\right\rangle \\
& \quad=\int_{\Sigma_{1}} \nabla_{T} u \nabla_{T} \theta+\Delta_{T} u \Delta_{T} \theta+u^{\prime} \theta^{\prime}+u \theta d s d t
\end{aligned}
$$

where $\int_{Q} \phi f d x d t$ means $\langle\cdot, \cdot\rangle_{L^{\infty}\left(0, T ; V^{\prime}\right), L^{1}\left(0, T ; H^{1}(\Omega)\right)}$. Next, we prove that

$$
\phi(T)=\rho_{0}, \quad \phi^{\prime}(T)=\rho_{1} .
$$

Let $\lambda$ be the eigenvalue for the $\Delta$ operator with mixed Wentzell, Dirichlet boundary conditions and $m$ be the corresponding eigenvector. The existence of eigenvalue for the $\Delta$ operator with mixed Wentzell, Dirichlet boundary condition is based on the fact that $\Delta^{-1}: L^{2}(\Omega) \rightarrow V$ is a compact operator. That is,

$$
\begin{cases}-\Delta m=\lambda m, & \text { in } \Omega, \\ \frac{\partial m}{\partial v}-\Delta_{T} m=0, & \text { on } \Gamma_{1}, \\ m=0, & \text { on } \Gamma_{0} .\end{cases}
$$

Set $f:=g(t) m$, where $g$ is a smooth function in $[0, T+\varepsilon]$, and let $\theta:=h(t) m$. Then

$$
\left\{\begin{array}{l}
h^{\prime \prime}+\lambda h=g, \\
h(0)=0, \quad h^{\prime}(0)=0 .
\end{array}\right.
$$

Claim $\exists g=g_{0}$ such that

$$
h(T)=h^{\prime}(T)=0, \quad h^{\prime \prime}(T) \neq 0 .
$$

If this is true, then

$$
\begin{aligned}
& \int_{Q} \phi g_{0}(t) m d x d t-\left\langle\rho_{1}, h(T) m\right\rangle+\left\langle\rho_{0}, h^{\prime}(T) m\right\rangle \\
& \quad=\int_{\Sigma_{1}}\left(\Delta_{T} u \Delta_{T} m-u^{\prime \prime} m+\nabla_{T} u \nabla_{T} m+m u\right) h(t) d s d t
\end{aligned}
$$


Since $h^{\prime \prime}+\lambda h=g_{0}$, we have

$$
\begin{gathered}
\int_{0}^{T}\left\langle\phi^{\prime \prime}+\lambda \phi, m\right\rangle h(t) d t+\langle\phi(T), m\rangle h^{\prime}(T)-\left\langle\phi^{\prime}(T), m\right\rangle h(T)+\left\langle\rho_{1}, m\right\rangle h(T)-\left\langle\rho_{0}, m\right\rangle h^{\prime}(T) \\
\quad=\int_{\Sigma_{1}} \Delta_{T} u \Delta_{T} m h(t)-u^{\prime \prime} m h(t)+\nabla_{T} u \nabla_{T} m h(t)+u m h(t) d s d t
\end{gathered}
$$

Differentiate (2.10) with respect to $T$, we get

$$
\begin{aligned}
\left\langle\phi^{\prime \prime}+\right. & \lambda \phi, m\rangle h(T)+\langle\phi(T), m\rangle h^{\prime \prime}(T)+\left\langle\phi^{\prime}(T), m\right\rangle h^{\prime}(T)-\left\langle\phi^{\prime \prime}(T), m\right\rangle h(T) \\
& -\left\langle\phi^{\prime}(T), m\right\rangle h^{\prime}(T)+\left\langle\rho_{1}, m\right\rangle h^{\prime}(T)-\left\langle\rho_{0}, m\right\rangle h^{\prime \prime}(T) \\
= & \int_{\Gamma_{1}}\left(\Delta_{T} u \Delta_{T} m-u^{\prime \prime} m+\nabla_{T} u \nabla_{T} m+u m\right) d s h(T) .
\end{aligned}
$$

Therefore

$$
\langle\phi(T), m\rangle h^{\prime \prime}(T)-\left\langle\rho_{0}, m\right\rangle h^{\prime \prime}(T)=0
$$

which implies that $\phi(T)=\rho_{0}$. Similarly, we obtain $\phi^{\prime}(T)=\rho_{1}$.

Now we prove the claim above. Write

$$
A:=\left(\begin{array}{ll}
0 & 1 \\
\lambda & 0
\end{array}\right), \quad B:=\left(\begin{array}{l}
0 \\
1
\end{array}\right)
$$

Then, by the Kalman condition [9], we know that (2.9) is controllable. Set $X(t):=$ $\left(h(t), h^{\prime}(t)\right)^{T}$. Then $\exists g_{1}(s), s \in\left(0, \frac{T}{2}\right)$, such that $X\left(\frac{T}{2}\right)=X_{0} \neq 0$. Write

$$
g_{2}\left(s-\frac{T}{2}\right):=B^{T} e^{A^{T}(T-s)} w^{-1}\left(-e^{A \frac{T}{2}} X_{0}\right),
$$

where $w=\int_{\frac{T}{2}}^{T} e^{A(T-s)} B B^{T} e^{A^{T}(T-s)} d s$. Then

$$
X(t)=e^{A\left(t-\frac{T}{2}\right)} X_{0}+\int_{\frac{T}{2}}^{t} e^{A(t-s)} B g_{2}\left(s-\frac{T}{2}\right) d s .
$$

Clearly, $X(T)=0, X^{\prime}(T) \neq 0$. This proof is then complete.

The following is our exact controllability theorem.

Theorem 2.3 Let $T>2 R$ and $F$ be the Hilbert space defined in (2.4). Then for every $\left(\phi^{\prime}(0),-\phi(0)\right) \in F^{\prime}$, there are $\left(u_{0}, u_{1}\right) \in F$ and a control function

$$
v(x, t)=-\partial_{t} u^{\prime}+\Delta_{T}\left(\Delta_{T} u\right)-\Delta_{T} u+u,
$$

where $u$ is the solution to (2.1), such that the solution $\phi(t)$ of system (2.5) with initial data $\left(\phi(0), \phi^{\prime}(0)\right)$ satisfies

$$
\phi(T)=0, \quad \phi^{\prime}(T)=0 .
$$


For the nonlinear case, we assume that $f \in W_{\text {loc }}^{1, \infty}(\mathbb{R})$ satisfies $f(0)=0$ and the superlinear condition (see [10]):

$$
\exists C>0, p>1: \quad\left|f^{\prime}(s)\right| \leq C|s|^{p-1}, \quad \forall s \in R \text { with } p<\frac{n}{n-\frac{6}{5}+\varepsilon} \text { if } n \geq 2 .
$$

Proposition 2.4 Assume that $f$ satisfies the super-linear condition (2.11). Then there exists $T_{0}>0$ such that for every $T>T_{0}$, there is a neighborhood $\omega$ of $(0,0)$ in $V \times L^{2}(\Omega)$ such that for each $\left(\phi_{0}, \phi_{1}\right) \in \omega$, there exists a control $\nu_{1} \in H^{-2}(\Gamma)$ such that the solution to (1.1) satisfies

$$
\phi(T)=0, \quad \phi^{\prime}(T)=0 .
$$

Proof From the results for the nonlinear system of Neumann problems (see [10]), we see that there exists a controllability $v \in L^{2}\left(\Gamma_{1}\right)$ such that the solution $\left(\phi, \phi^{\prime}\right)$ of the following system:

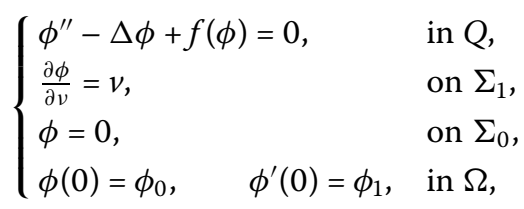

satisfies $\left(\phi(T), \phi^{\prime}(T)\right)=(0,0)$, and $\phi \in H^{\beta}(\Omega)$ where $\beta \leq \frac{3}{5}-\varepsilon$. The regularity of $\phi$ for Neumann problems can be found in Theorem 1.4 of [11]. Let $v_{1}=v-\Delta_{T} \phi$. Then

$$
\frac{\partial \phi}{\partial v}-\Delta_{T} \phi=v_{1}
$$

and $v_{1} \in H^{-2}\left(\Gamma_{1}\right)$ such that $\phi(T)=0, \phi^{\prime}(T)=0$.

Remark 2.1 For dynamic Wentzell systems with boundary condition (1.2), we can also prove the results as Theorem 2.3 and Proposition 2.4 by similar arguments.

\section{Competing interests}

The authors declare that they have no competing interests.

Authors' contributions

Both authors contributed equally and significantly in writing this paper. Both authors read and approved the final manuscript.

\section{Acknowledgements}

Ti-Jun Xiao acknowledges support from NSFC (Nos. 11271082, 11371095).

Received: 5 November 2015 Accepted: 27 January 2016 Published online: 05 February 2016

\section{References}

1. Lemrabet, K: Le problème de Ventcel pour le systeme de l' élasticité dans un domainé de $R^{3}$. C. R. Acad. Sci. Paris, Sér. I Math. 304(6), 151-154 (1987)

2. Cavalcanti, M, Lasiecka, I, Toundykov, D: Wave equation with damping affecting only a subset of static Wentzell boundary is uniformly stable. Trans. Am. Math. Soc. 364(11), 5693-5713 (2012)

3. Heminna, A: Contrôlabilité exacte d'un problème avec conditions de Ventcel évolutives pour le système linéaire de l'élasticité. C. R. Acad. Sci. Paris, Sér. I Math. 324(2), 195-200 (1997)

4. Tong, C, Wang, YD: Existence of solutions for an initial control problem with dynamic boundary conditions. J. Shanghai Univ. Nat. Sci. 20(6), 741-748 (2014)

5. Xiao, TJ, Liang, J: Complete second order differential equations in Banach spaces with dynamic boundary conditions. J. Differ. Equ. 200, 105-136 (2004) 
6. Xiao, TJ, Liang, J: Second order differential operators with Feller-Wentzell type boundary conditions. J. Funct. Anal. $254,1467-1486(2008)$

7. Xiao, TJ, Liang, J: Nonautonomous semilinear second order evolution equations with generalized Wentzell boundary conditions. J. Differ. Equ. 252, 3953-3971 (2012)

8. Tucsnak, M, Weiss, G: Observation and Control for Operator Semigroups. Birkhäuser Advanced Texts: Basler Lehrbücher. Birkhäuser, Basel (2009)

9. Coron, JM: Control and Nonlinearity. Mathematical Surveys and Monographs, vol. 136. Am. Math. Soc., New York (2007)

10. Zuazua, E: Exact controllability for the semilinear wave equation. J. Math. Pures Appl. 69(9), 1-31 (1990)

11. Lasiecka, I, Triggiani, R: Sharp regularity theory for second order hyperbolic equations of Neumann type. Ann. Mat. Pura Appl. 157(1), 285-367 (1990)

Submit your manuscript to a SpringerOpen ${ }^{\circ}$ journal and benefit from:

- Convenient online submission

Rigorous peer review

- Immediate publication on acceptance

- Open access: articles freely available online

- High visibility within the field

- Retaining the copyright to your article 